\title{
The prognosis of the diabetic retinopathy using computer science and biotechnology
}

\author{
Irina Vorobyeva ${ }^{1, *}$ \\ ${ }^{1}$ Russian Medical Academy of Continuous Professional Education, 2/1, Barrikadnaya Street, \\ Moscow, 125993, Russia.
}

\begin{abstract}
The problem of diabetic retinopathy causes loss of the vision. In nowadays, modern equipment is used to diagnose this disease: an optical coherent tomograph and a fundus microperimeter. For even more accurate diagnosis, biomarker levels in the lacrimal fluid and blood serum are being researched. The mathematical model for predicting the developing of the course of the disease is proposed. The mathematical model is based on four mathematical criteria. The first two criteria analyze the data of digital equipment: the thickness of the retina in 9 zones and the photosensitivity of the macula. The third and the fourth mathematical criteria analyze the levels of pathogenetic biomarkers in the lacrimal fluid (vascular endothelial growth factor VEGF-A) and the blood serum (glycated hemoglobin). The program based on the proposed mathematical formulas combines the objective data from the digital equipment and the biomarkers. The program gives the possibility for the automatic prediction of developing the diabetic retinopathy. The prognosis of development of the course of the disease according to the program coincides with a clinical prognosis of a doctor. It was shown that the application of the proposed complex approach gives more opportunities in comparison with separate studies of the biomarkers, morphology and retinal function.
\end{abstract}

\section{Introduction}

\subsection{The problem of diabetic retinopathy in medicine}

The problems of diabetes and diabetic retinopathy causes loss of the vision [1, 2]. Late diagnosis of the disease leads to blindness [3].

The search for biotechnologies [4, 5], biomarkers [6-10] in blood serum and lacrimal fluid for prognosis of the disease is important for early diagnosis of the diabetic retinopathy [11]. Various scientists have been investigating such significant biomarkers as the vascular endothelial growth factor (VEGF-A) [12], monocytic chemoattractant protein (MCP-1) [13], matrix metalloproteinases (MMP-2, MMP-9) [14]. A lot of researches are devoted to genetic mutations of diabetes patients [15].

\footnotetext{
*Corresponding author: irina.docent2000@mail.ru
} 
Early detection of the disease allows to select the right treatment method. The effectiveness of using antiangiogenic drugs, laser and surgical treatments is being investigated all over the world [16-18].

\subsection{Computer software and equipment are used to diagnose diabetic retinopathy}

Researchers use a variety of diagnostic equipment to diagnose the diabetic retinopathy, such as an optical coherent tomograph [19], fundus photo recording, fundus microperimeter [20].

The optical coherent tomograph is a device which allows to determine the structure of the retina. The operation of the device is based on the physical phenomenon of interference. The image received from the device is automatically transferred to a computer, which gives an opportunity to visualize the layers of the retina on a monitor screen.

The fundus microperimeter gives an opportunity to determine the photosensitivity of the retina and its' thresholds. The operation of the device is based on the method of neural networks. The sensory image on the device screen gives an information about the fundus image, histogram, light sensitivity thresholds and a color analysis scale with 37 points in the center of the retina.

The described devices give an opportunity to make a diagnosis at an early stage of the disease, because the devices analyze the morphology and the function of the retina. A significant advantage of this approach is the ability to diagnose the diabetic retinopathy at an early stage, which cannot be checked by an ophthalmologist using the classic fundus ophthalmoscopy method.

The diagnosis of the diabetic retinopathy can be confirmed by studying the levels of biomarkers in blood serum and in lacrimal fluid. The advantage of biomarkers studying is the ability of determining the deviation of patient's biomarkers levels from norm, which enables doctors to diagnose the disease, even if the ophthalmic equipment does not detect changes in the fundus.

\section{Problem}

Thus, the combination of using the biomarkers and the diagnostic equipment is an efficient way of the early detection of the diseases. However, this approach does not give an opportunity to determine the risk of development of the disease and its prognosis.

The aim of this work is developing a mathematical model for predicting the disease using biomarkers, data of ophthalmic equipment and creating a computer program.

\section{Materials and methods}

\subsection{Mathematical processing of objective digital data of an optical coherent tomograph}

Map of the macula E MM5 (Fig. 1) and a table of thicknesses of the retina of the macula (Fig. 2) are the results of the operation of an optical coherent tomograph.

A criterion of the influence of retinal thickness, which is characterized by the severity of the volume of edema in the thickness of the retina in 9 sectors of the macular zone, is proposed: 


$$
R_{1}=\frac{\sum_{1}^{9}\left(\mathrm{~h}_{i}-\mathrm{h}_{\text {min }}\right)}{\left(\mathrm{h}_{\text {max }}-\mathrm{h}_{\text {min }}\right) \cdot \mathrm{m}} .
$$

where $R_{1}$ - retinal thickness criterion;

$\mathrm{h}_{i}$ - thickness of retinal sectors (in each sector of the macular zone), $\mu \mathrm{m}$;

$\mathrm{h}_{\min }$ - retinal thickness in one of the scanning sectors of the macular zone, excluding the central and extreme sectors, $\mu m$;

$\mathrm{h}_{\max }-$ maximum retinal thickness of the macular zone, $\mu \mathrm{m}$;

$\mathrm{m}$ - number of retinal scan sectors $(\mathrm{m}=9)$.



Fig. 1. Map of the macula E MM5 with an information about the thickness of the retina of the macula in fovea, parafovea, perifovea according to the optical coherent tomograph, $\mu \mathrm{m}$.

\begin{tabular}{|l|l|l|}
\hline Section & Thick $(\mu \mathrm{m})$ & Vol $\left(\mathrm{mm}^{3}\right)$ \\
\hline Fovea & 390 & 0.306 \\
\hline ParaFovea & 375 & 2.354 \\
\hline - S. Hemisphere & 392 & 1.233 \\
\hline I. Hemisphere & 357 & 1.121 \\
\hline Tempo & 423 & 0.664 \\
\hline - Superior & 382 & 0.610 \\
\hline Nasal & 356 & 0.559 \\
\hline Inferior & 332 & 0.521 \\
\hline Perifovea & 325 & 4.086 \\
\hline S. Hemisphere & 333 & 2.092 \\
\hline I. Hemisphere & 317 & 1.994 \\
\hline Tempo & 302 & 1.231 \\
\hline Superior & 320 & 1.004 \\
\hline Nasal & 306 & 0.962 \\
\hline Inferior & 283 & 0.889 \\
Yol within: 0.306(1 & $2.660(3 \mathrm{~mm})$ & $6.747(6 \mathrm{~mm})$ \\
\hline
\end{tabular}

Fig. 2. Table of retinal thicknesses of the macula in fovea, parafovea, perifovea according to the optical coherent tomograph, $\mu \mathrm{m}$. 


\subsection{Mathematical Processing of Objective Digital Data from the MAIA Fundus Microperimeter}

Sensitivity map (Fig. 3) and histogram of threshold frequencies (Fig. 4) are the results of the fundus microperimeter.

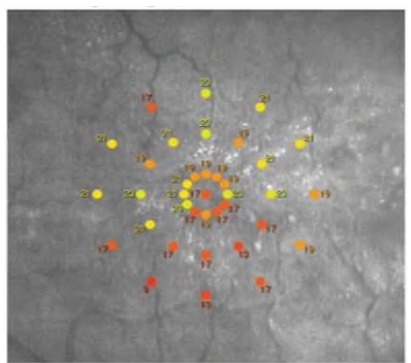

Fig. 3. Sensitivity map.

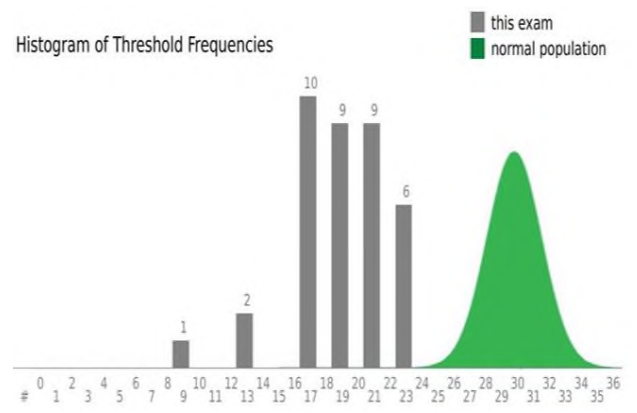

Fig. 4. Histogram of threshold frequencies.

A criterion of the influence of photosensitivity, which characterizes the degree of change in the sensitivity thresholds of the macular zone of the retina taking into account the intensity of the light stimulus, is proposed:

$$
R_{2}=\frac{\sum_{1}^{36}\left(I_{i}-I_{\text {min }}\right)}{\left(I_{\max }-I_{\min }\right) \cdot 36} .
$$

where $R_{2}$ - sensitivity criterion;

$I_{i}-$ light stimulus intensity, $d b$;

$I_{\text {min }}$ - minimum light stimulus intensity, $d b$;

$I_{\text {max }}$ - maximum light stimulus intensity, $d b$;

36 - the number of points at which the light stimuli are determined.

\subsection{Mathematical processing of an objective indicator of serum biomarker level}

It is proposed to take the level of glycated hemoglobin as the main serum blood biomarker describing the degree of compensation of diabetes mellitus. A criterion of the influence of glycated hemoglobin, which describes the nature of the relationship between the state of the 
morphological structures of the retina, corresponding to the severity of the volume of edema according to the value of the criterion and the degree of compensation of diabetes mellitus, is proposed:

$$
R_{3}=\frac{G}{G_{c r i}}
$$

where $R_{3}$ - glycated hemoglobin effect criterion;

$G$ - glycated hemoglobin level from the studied patient, \%;

$G_{c r i}$ - upper limit of glycated hemoglobin level, \%.

\subsection{Mathematical processing of an objective indicator of the biomarker level in the lacrimal fluid}

It is proposed to use the vascular endothelial factor (VEGF-A) as the main biomarker in the lacrimal fluid, which describes the pathogenetic mechanisms in the eye with the diabetic retinopathy.

A criterion of the influence of the level of vascular endothelial growth factor, describing the relationship between the state of the morphological structures of the retina and the level of vascular endothelial growth factor in the lacrimal fluid, is proposed:

$$
R_{4}=\frac{V E G F}{V E G F_{\max }} .
$$

where $R_{4}$ - criterion of the influence of the vascular endothelial growth factor level;

$V E G F$ - level of vascular endothelial growth factor in the lacrimal fluid from the studied patient, $p g / m l$;

$V E G F_{\max }-$ maximum level of endothelial growth factor, $\mathrm{pg} / \mathrm{ml}$.

\subsection{A mathematical model for predicting disease progression}

The developing formulas (1) - (4) are based on the mathematical method of normalizing the studying value. In the diagnosis of the diabetic retinopathy, it is proposed that all digital data can be divided into 4 factors which affect the diagnosis and prognosis of the disease ( $R_{1}$ - the influence of retinal thickness; $R_{2}$ - the influence of sensitivity; $R_{3}$ - the influence of the level of glycated hemoglobin; $R_{4}$ - the influence of the level of vascular endothelial growth factor).

All calculated criteria are combined in one integral criterion of progression of the disease:

$$
R_{\text {general }}=R_{1} \cdot R_{2} \cdot R_{3} \cdot R_{4}
$$

where $R_{\text {general }}$ - integral criterion of progression of the disease.

The boundary value table for the prognosis of diseases (table 1) was developed on the basis of statistical data processing. 
Table 1. The prognosis of the disease depending on the criterion $R$

\begin{tabular}{|c|c|c|}
\hline$R_{\text {general }}$ & Disease diagnosis & Disease prognosis \\
\hline$R_{\text {general }} \leq 0.07$ & $\begin{array}{c}\text { Non-proliferative stage of } \\
\text { diabetic retinopathy with } \\
\text { diabetic macular edema }\end{array}$ & $\begin{array}{c}\text { Low risk of disease } \\
\text { progression with a good } \\
\text { prognosis for vision }\end{array}$ \\
\hline$R_{\text {general }} \geq 0.18$ & $\begin{array}{c}\text { Preproliferative stage of } \\
\text { diabetic retinopathy with } \\
\text { diabetic macular edema }\end{array}$ & $\begin{array}{c}\text { High risk of disease } \\
\text { progression with a bad } \\
\text { prognosis for vision }\end{array}$ \\
\hline $\begin{array}{c}\text { Proliferative stage of } \\
\text { diabetic retinopathy with } \\
\text { diabetic macular edema }\end{array}$ & $\begin{array}{c}\text { High risk of disease } \\
\text { progression with a bad } \\
\text { prognosis for vision }\end{array}$ \\
\hline
\end{tabular}

\section{Results and discussion}

Based on the proposed mathematical model, a computer program was developed, which gives the possibility to predict the prognosis of the course of the disease. The initial data for the program operation are 4 parameters: a retinal thickness map in the macular zone $\mathrm{E}$ MM5 (Fig. 1), macula photosensitivity (Fig. 3), level of a pathogenetic biomarker in blood serum (glycated hemoglobin), level of a pathogenetic marker in lacrimal fluid (endothelial growth factor VEGF-A vessels). The result of the program is the automatic prediction of the course of the disease (table 1).

The program was used to determine the prognosis of course of the disease for the patient, and the results of its work were compared with the doctor's clinical prognosis of the course of the disease.

The patient examination data are presented by an optical coherent tomograph (table 2).

Table 2. Retinal thickness of the examined patient in the macula (fovea, parafovea, perifovea) according to the optical coherence tomograph data.

\begin{tabular}{|c|c|c|}
\hline \multicolumn{2}{|c|}{ Section } & Thick, $\mu \mathrm{m}$ \\
\hline \multicolumn{2}{|c|}{ Fovea centralis } & 390 \\
\hline \multirow{4}{*}{ ParaFovea } & Tempo & 423 \\
\cline { 2 - 3 } & Superior & 389 \\
\cline { 2 - 3 } & Nasal & 356 \\
\cline { 2 - 3 } & Inferior & 332 \\
\hline \multirow{4}{*}{ PeriFovea } & Tempo & 392 \\
\cline { 2 - 3 } & Superior & 320 \\
\cline { 2 - 3 } & Nasal & 306 \\
\cline { 2 - 3 } & Inferior & 283 \\
\hline
\end{tabular}

Patient examination data are presented by the MAIA fundus microperimeter (table.3).

Table 3. Retinal light stimulus of the macula according to the MAIA fundus microperimeter data.

\begin{tabular}{|c|c|}
\hline $\begin{array}{c}\text { Intensity of light } \\
\text { stimulus, } d b\end{array}$ & $\begin{array}{c}\text { The number of points } \\
\text { with a particular intensity }\end{array}$ \\
\hline 9 & 1 \\
\hline 13 & 2 \\
\hline 17 & 10 \\
\hline 19 & 9 \\
\hline 21 & 9 \\
\hline 23 & 6 \\
\hline
\end{tabular}


Laboratory data is presented for determining the level of the biomarkers for the patient in blood serum and lacrimal fluid (table. 4).

Table 4. Laboratory data for determining the level of biomarkers for the patient in blood serum and lacrimal fluid.

\begin{tabular}{|c|c|}
\hline $\begin{array}{c}\text { Level of serum glycated } \\
\text { hemoglobin, \% }\end{array}$ & $\begin{array}{c}\text { Level of vascular } \\
\text { endothelial growth factor } \\
\text { (VEGF-A) in the } \\
\text { lacrimal fluid, } \mathrm{pg} / \mathrm{ml}\end{array}$ \\
\hline 7.2 & 800 \\
\hline
\end{tabular}

The result of calculations of the program is determining the value of the integral criterion of progression $R_{\text {general }}=0.02$ and automatic prediction of the course of the disease. This individual patient prognosis is "low risk of disease progression with a good prognosis for vision" (table 1).

The risk of the disease progression, which was obtained in automatic mode, coincides with the doctor's medical report according to the proposed mathematical model.

Thus, the proposed mathematical method gives the opportunity to determine the risk of disease progression based on objective morphological criteria according to the data of an optical coherent tomograph, on objective functional indicators of the photosensitivity of the macula according to fundus microperimeter and on an objective analysis of laboratory biomarkers in the lacrimal fluid and the blood serum.

The proposed mathematical method gives more opportunities in comparison with the traditional individual methods of investigating biomarkers in the lacrimal fluid and the blood serum, or with morphology analysis and function according to optical coherent tomograph.

\section{Conclusion}

The mathematical model was proposed for the prognosis of the course of the diabetic retinopathy, which has combined objective morphological data of an optical coherent tomograph, photosensitivity of the macula according to the fundus microperimeter and objective indicators of the biomarkers in the lacrimal fluid and in the blood serum.

A mathematical model was created to simplify the analysis of huge digital material and to systematize the data of an optical coherent tomograph and microperimeter for patients with diabetic retinopathy.

A complex approach for the mathematical model gives the possibility to predict the pathogenetic level of diabetic retinopathy.

The described approach to the prognosis of the course of the diabetic retinopathy using a mathematical model gives an opportunity to make a personalized approach to the patient taking into account the complex of his criterion which can be calculated by the proposed formulas.

The proposed program gives a possibility for a doctor to analyze the results of the examination automatically.

The created effective mathematical models made it possible to reach an interdisciplinary level using exact mathematics and digital technologies for quantitative and qualitative assessment of the structures of the fundus.

The criteria obtained by the formulas coincide with the results of the clinical diagnosis and assessment of fundus structures. 


\section{References}

1. M. Mutambudzi, C. Gonzalez and R. Wong(2020) Impact of Diabetes and Disease Duration on Work Status Among US Older Adults, Journal of aging and health, $32 \mathrm{~L}$ 5-6, 432-40

2. E. Kucuk, Zor K and Yilmaz U Causes of Blindness and Moderate to Severe Visual Impairment in Nigde, Central Anatolia, Turkey, Erciyes medical journal, 41 L 4, 42024 (2019)

3. Z. Yasir, A. Hassan and K. Rajiv Diabetic retinopathy (DR) among 40 years and older Saudi population with diabetes in Riyadh governorate, Saudi Arabia - A population based survey, Saudi journal of ophthalmology. 33 L 4, 363-8 (2019)

4. A. Dinnyes, A. Schnur, S. Muenthaisong Integration of nano- and biotechnology for beta-cell and islet transplantation in type-1 diabetes treatment, Cell proliferation. L e12785 (2020)

5. M. Ahmed, M. Islam, B. Paul, Computational modeling and analysis of gene regulatory interaction network for metabolic disorder: a bioinformatics approach, Biointerface research in applied chemistry. 10 L 4, 5910-17 (2020)

6. H. L. Deissler,G.K. Lang Inhibition of Single Routes of Intracellular the Biphasic Disturbance of a Retinal Endothelial Cell Barrier Induced by VEGF-A(165), Cellular physiology and biochemistry, 42 L 4, 1493-13 (2017)

7. H. Chen, X.Z. Zhang, N.Y. Liao The moisture regime calculation of single-layer enclosing structures on the basis of the discrete-continuum method application, $B M C$ ophthalmology. 17, 176 (2017)

8. M.W. Stewart A review of ranibizumab for the treatment of diabetic retinopathy, Ophthalmology and Therapy. 6, 33-47 (2017)

9. I. Waltl, C. Zehetner, C. Seifarth, Effects of Intravitreal Aflibercept on Galectin-1 and Vascular Endothelial Growth Factor-A Plasma Levels in Patients with Diabetic Retinopathy, Current eye research. 43, 368-75 (2018)

10. A. Xiao, Q. Zhou, Y. Shao CFD modeling of convective scalar transport in a macroporous material for drying applications, Experimental and therapeutic medicine, 13, 3360-68 (2017)

11. S. Arf, I.S. Muslubas, M. Hocaoglu Spectral domain optical coherence tomography classification of diabetic macular edema: a new proposal to clinical practice, Graefes archive for clinical and experimental, 258, 1165-72 (2020)

12. H.L. Deissler, L.N. Stutzer, G.K. Lang VEGF receptor 2 inhibitor nintedanib completely reverts VEGF-A(165)-induced disturbances of barriers formed by retinal endothelial cells or long-term cultivated ARPE-19 cells, Experimental eye research. 194, L 108004 (2020)

13. S.Y. Yu, D. H. Nam Changes in aqueous concentrations of various cytokines after intravitreal bevacizumab and subtenon triamcinolone injection for diabetic macular edema, Graefes archive for clinical and experimental ophthalmology, 256 L 1, 39-47 (2018)

14. J.H. Wang, D.M. Ling, L.L. Tu, Boguszewska-Czubara A, Tylus M and SwiechZubilewicz A, Life sciences. 229, 149-56 (2019)

15. J.H. Wang, D.M. Ling, L.L. Tu, therapy for diabetic retinopathy: Are we ready to make the leap from bench to bedside, Pharmacology and therapeutics, 173, 1-18 (2017) 
16. I.V. Vorobyeva, L.K. Moshetova Therapy results in patients with primary open angle glaucoma and diabetic retinopathy, Ophthalmology in Russia, 14 L 1, 40-46 (2017)

17. I.V. Vorobyeva Long-term results of treatment of patients with non-proliferative diabetic retinopathy angioprotectors, Ophthalmology in Russia, 14, L 1, 67-77 (2017)

18. I.V. Vorobyeva Monitoring of separate pathogenetically significant biochemical markers in lacrimal fluid, ophthalmological parameters with combined pathology of diabetic retinopathy and age-related macular degeneration on the background angioprotective and antioxidant therapy Ophthalmology in Russia. 15, L 2,189-99 (2018)

19. J.W. Wang, C.H. Jie Macular integrity assessment to determine the association between macular microstructure and functional parameters in diabetic macular edema International journal of ophthalmology. 11 L 7, 1185-91 (2018)

20. S. N. Markowitz and S.V. Reyes Microperimetry and clinical practice: an evidencebased review Canadian journal of ophthalmology-journal canadien d ophtalmologie. 48 L 5, 350-57 (2018) 